\title{
ANALYSIS AND OPTIMIZATION OF BER FOR SECONDARY USERS IN COGNITIVE RADIO USING BBO AND PSO METHODS
}

\author{
Atul Goyal ${ }^{1}$, Ashish Raman ${ }^{2}$ \\ ${ }^{1} M$. Tech Scholar, ECE Department, Dr B R Ambedkar NIT, Jalandhar, India \\ ${ }^{2}$ Assistant Professor, ECE Department, Dr B R Ambedkar NIT, Jalandhar, India
}

\begin{abstract}
Cognitive radio has been recognized as an answer to problem of limited spectrum in wireless communication. In this paper, the Bit-error-rate (BER) of secondary users in Cognitive radio network has been reduced using the Biography-based optimization (BBO) and Particle swarm optimization (PSO) techniques by maximizing the channel gains. For each secondary transmitter antenna, the channel gain for secondary to secondary link and secondary to primary link is optimized and the data is sent from an antenna using constrained difference selection method, showing maximum gain. Other than selection of antenna, another constraint for the optimization is the transmit power for secondary user. The BER has been improved for various values of Signalto-noise ratio (SNR) over which the transmission took place. It is proved from the simulation results that the average BER has been reduced by $8.571 \%$ and $5.229 \%$ with BBO and PSO respectively. It has been shown that BBO and PSO have a trade-off between reduction of average SNR and Time elapsed. BBO takes 0.048169 seconds for calculation whereas PSO does the same in 0.036195 seconds. At last, practical applications of both techniques are discussed.
\end{abstract}

Keywords: Cognitive radio, BBO, PSO, BER, secondary user.

\section{INTRODUCTION}

Wireless communication is an expanding field where new technologies are being developed and enhanced every day. With the growing rate of new consumers, the demand for more bandwidth is increasing; however, the usable spectrum size is constant [1]. While new technologies are present that are working on higher frequency range like $3 \mathrm{G}, 4 \mathrm{G}$ in cellular applications [2], the complete spectrum is still not used effectively. At a time, when some part of the spectrum is heavily occupied, but other part is used intermittently [3]. This situation can be exploited by allowing the Secondary users to use the spectrum without causing any interference to the primary user. But the constraints on secondary user are transmit power of secondary transmitter antennas and difference selection weight [4], in order to achieve diversity. After setting the aforementioned constraints, the objective of this work is to minimize the BER for secondary transmitters. The key to this lies in the selection of antenna from which the data is to be transmitted. One of the methods to do so is by using difference antenna selection [5]. In this method a subset of antennas is used for the transmission of data, instead of using all the available antennas. The value of difference selection weight $(\delta)$ lies in the range [0 - 1], where full diversity is achieved for $\delta=1$ [6]. For each antenna, the channel gains are optimized by using the BBO and PSO techniques [7], [8] and difference selection weight is used to employ diversity in transmission [9]. The rest of the paper is organized as follows. Section 2 describes the Cognitive radio networks viz. BBO, PSO methods and the formulation of problem. However, the application of optimization techniques in CR domain for reducing the BER with different parameters has been explained in Section 3 . Section 4 shows the simulation results of the proposed work.
Finally, a conclusion is given by section 5, describing the practical application of optimized BER with both methods.

\section{SYSTEM MODEL AND PROBLEM FORMULATION}

\subsection{Cognitive Radio Network}

A Cognitive radio (CR) system is considered having a single primary link and a secondary link. The CR system is configured with $\mathrm{M}=4$ secondary transmitters and one secondary receiver [6]. The constraint on the $i^{\text {th }}$ antenna for secondary transmitter is that, it should send the data with less than threshold power of primary receiver [10].

$$
E \mathrm{~s}\left(\overline{\mathrm{y}}_{\mathrm{p}, \mathrm{i}}\right) \leq \wp
$$

Where $\wp$ is the average interference threshold at the primary receiver, $\mathbb{E}$ stands for expectation operator, $E_{\mathrm{s}}$ is average secondary transmit power.

The objective of this work is to minimize the average BER for secondary transmitter defined as

$$
\operatorname{minimize} \mathcal{P}_{\mathrm{b}}(E S, \delta)
$$

where $\mathcal{P}_{\mathrm{b}}$ is the uncoded BER of secondary transmitter and $\delta$ is the difference selection weight.

The idea is to optimize the gain from secondary to secondary link and secondary to primary link denoted as $\gamma_{\mathrm{s}, \mathrm{i}}$ and $\gamma_{\mathrm{p}, \mathrm{i}}$ respectively. The probability density function (p.d.f.) of the $\gamma_{\mathrm{s}, \mathrm{i}}$ and $\gamma_{\mathrm{p}, \mathrm{i}}$ are given as 




Respectively where $\bar{y}_{\mathrm{s}}$ and $\bar{y}_{\mathrm{p}}$ are the corresponding average channel gain

\subsection{Biography Based Optimization}

Biography- based optimization is based on the evolution and migration of new species [7]. All the species migrate from one island to another. Each island has its own habitat suitability index (HSI). The HSI depends upon various factors like atmosphere, weather, temperature and others [2]. An island having high value of HSI has more number of species living on it. Species emigrate from their host island to other because of the large number of population due to other species. An island with high HSI has high emigration rate and low immigration rate. Similarly species living on an island with low HSI immigrate to other island with high HSI in search of better circumstances to grow [11]. So an island with low HSI has high immigration rate and low emigration rate. After a pre-defined number of iterations, the best resultant island is considered as the optimized solution for given fitness function [12].

\subsection{Particle Swarm Optimization}

The Particle swarm optimization is a technique to optimize a problem by computing and refining the candidate solution iteratively for a particular fitness function in any domain [8]. The initial population is taken within the restrained searchspace for solutions which is defined according to the problem in hand. The candidate solutions or swarm particles travel in the search-space with their resultant velocity [13]. The Resultant velocity is a vectored combination of the particle's previous position, particle's previous best position and swarm's best position [14]. With each iteration, the swarm's best position is updated which leads to the optimized resultant solution for the given fitness function [12].

\section{METHODOLOGY}

\subsection{BER Analysis}

The CR network has been considered with BPSK modulation for simplicity. Additive white Gaussian noise is considered for the wireless fading channel.

Step 1: Following denotations are used for the calculation of BER [4]:

$$
\begin{aligned}
& \varpi_{s}=(1-\delta) \bar{y}_{s}, \varpi_{p}=\delta \bar{y}_{p} \text { andy }=\varpi_{s}+\varpi_{p} \\
& \mathcal{P}_{b}(\delta, y)= \begin{cases}\frac{M}{2 \bar{y}_{s}} \boldsymbol{\Phi}(\delta, y) & , \delta=1 \\
\frac{1}{2} \sum_{k=0}^{M-1} \boldsymbol{\Psi}_{k}(\delta, y)+\frac{\varpi_{p}^{M-1}}{2 \bar{y}^{M-1} \bar{y}_{s}} \boldsymbol{\Theta}(\delta, y)+\frac{M \delta}{2 \bar{y}} \boldsymbol{\Phi}(\delta, y) & , \delta \neq 1, \varpi_{s} \neq \mathrm{g}_{p} \\
\frac{1}{2} \sum_{\substack{k=0 \\
k \neq g}}^{M-1} \boldsymbol{\Psi}_{k}(\delta, y)+\frac{\varpi_{p}^{M-1}}{2 \bar{y}^{M-1} \bar{y}_{s}} \boldsymbol{\Theta}(\delta, y)+\frac{M \delta}{2 \bar{y}} \boldsymbol{\Phi}(\delta, y)+\frac{M \delta}{2 \bar{y}} \boldsymbol{\Upsilon}_{\boldsymbol{g}}(\delta, y), \delta \neq 1, \varpi_{s}=\mathrm{g \varpi}_{p}\end{cases}
\end{aligned}
$$

$$
\overline{\mathrm{y}}=E_{b} / N_{0},
$$

Where $E_{b}$ is the transmitted power and $N_{0}$ is the noise power.

Step 2: Using the denotations in Step 1, the following functions to be used in the derivation of optimizing problem are defined as [6]

$$
\begin{gathered}
\boldsymbol{\Theta}(\delta, y)=\frac{\varpi_{s} \varpi_{p}}{\delta \overline{\mathrm{y}}}\left(1-\sqrt{\frac{\overline{\mathrm{y}}_{s} \varpi_{p} y}{\overline{\mathrm{y}}_{s} \varpi_{p} y+\overline{\mathrm{y}}}}\right)(1) \\
\Omega_{k}(y)=\frac{\bar{y}_{s}}{k+1}\left(1-\sqrt{\frac{\overline{\mathrm{y}}_{s} y}{\overline{\mathrm{y}}_{s} y+k+1}}\right) \\
\Gamma_{g}(y)=\left(\frac{\bar{y}_{s}}{g+1}\right)^{2}\left[1-\sqrt{\overline{\mathrm{y}}_{s} y} \frac{\overline{\mathrm{y}}_{s} y+3(g+1)}{\left(\overline{\mathrm{y}}_{s} y+(g+1)\right)^{3 / 2}}\right]
\end{gathered}
$$

Step 3: With the help of equation (2), the channel gain for secondary to secondary link is defined as [15],

$$
\begin{aligned}
\boldsymbol{\Phi}(\delta, y)= & \int_{0}^{\infty} \operatorname{erfc}(\sqrt{\delta x}) \phi(x) d x \\
& =\sum_{k=0}^{M-1}\left(-\frac{\sigma_{s}}{\bar{y}}\right)^{k} \Omega_{k}(y)
\end{aligned}
$$

where $\phi(x)$ is the p.d.f. for $s \rightarrow s$ link.

Step 4: Inferring equation (1) and (2), the channel gain secondary to primary link is defined as [15],

$$
\begin{aligned}
\boldsymbol{\Psi}_{\boldsymbol{k}}(\delta, y) & =\int_{\mathbf{0}}^{\infty} \operatorname{erfc}(\sqrt{y x}) \psi(x) d x \\
& =M \sum_{k}^{M-1} \frac{-\varpi_{s}{ }^{k+1} \varpi_{p}}{\overline{\mathrm{y}}^{k+1}\left(\varpi_{s}-k \varpi_{p}\right)} \\
& X\left(-\frac{k+1}{\bar{y}_{S}} \Omega_{k}(y)+\frac{\delta \overline{\mathrm{y}}}{\varpi_{s} \varpi_{p}} \boldsymbol{\Theta}(\delta, y)\right)
\end{aligned}
$$

where $\psi(x)$ is p.d.f. for $s \rightarrow p$ link.

Step 5: Using equation (2) and (3), the channel gain for primary to primary link is considered as [15],

$$
\begin{array}{r}
\mathbf{\Upsilon}_{\boldsymbol{g}}(\delta, y)=\int_{\mathbf{0}}^{\infty} \operatorname{erfc}(\sqrt{y x}) \mathrm{v}(g, x) d x \\
=\sum_{g}^{M-1}\left(-\frac{\omega_{s}}{\bar{y}}\right)^{g} \frac{g+1}{\bar{y}_{s}} \Gamma_{g}(y) \\
\quad-\sum_{g}^{M-1}\left(-\frac{\varpi_{s}}{\bar{y}}\right)^{g} \Omega_{g}(y)
\end{array}
$$

where $v(g, x)$ is the p.d.f. for $p \rightarrow p$ link.

Step 6: From equations (1), (4), (5) and (6) above, the BER for secondary transmitter is derived as 


\subsection{Optimization}

For $\mathrm{BBO}$ technique, initially 80 candidate solutions are taken and the channel gains are calculated with equation (4), (5) and (6) for each solution within the specified range as given in Table-1. Mutation factor of 0.05 is used to upgrade the properties of candidate solutions to get the optimized value of BER in 50 iterations. Table- 1 descripts the rest of the parameters used in the optimization technique.

Table-1: Parameters used in proposed work for Biography based Optimization

\begin{tabular}{|l|l|}
\hline Parameters & Value \\
\hline Initial Population & 80 \\
\hline Number of Iterations & 50 \\
\hline Probability of Mutation & 0.05 \\
\hline Number of Elite Solutions & 2 \\
\hline Number of Antennas & 4 \\
\hline $\begin{array}{l}\text { Minimum value of Difference } \\
\text { Selection weight }\end{array}$ & 0 \\
\hline $\begin{array}{l}\text { Maximum value of Difference } \\
\text { Selection weight }\end{array}$ & 1 \\
\hline $\begin{array}{l}\text { Minimum value of Secondary } \\
\text { transmit Power }\end{array}$ & $0 \mathrm{~dB}$ \\
\hline $\begin{array}{l}\text { Maximum value of Secondary } \\
\text { transmit Power }\end{array}$ & $20 \mathrm{~dB}$ \\
\hline
\end{tabular}

For PSO method, 80 swarm particles are taken initially. For each particle or candidate solution the fitness function is calculated using equation (7). Iteratively, the combined result of best available solution, previous best solution and previous solution are taken to calculate the next value. If the resultant is better than the best available solution, then its value is stored in the best available solution. The rest of the parameters are given in Table- 2 .

Table-2: Parameters used in proposed work for Particle Swarm Optimization

\begin{tabular}{|l|l|}
\hline Parameters & Value \\
\hline Initial Population & 80 \\
\hline Number of Iterations & 50 \\
\hline Number of Antennas & 4 \\
\hline Convergence factor & 0.2 \\
\hline$\varphi_{1}$ and $\varphi_{2}$ & 0.8 \\
\hline $\begin{array}{l}\text { Minimum value of Difference } \\
\text { Selection weight }\end{array}$ & 0 \\
\hline $\begin{array}{l}\text { Maximum value of Difference } \\
\text { Selection weight }\end{array}$ & 1 \\
\hline $\begin{array}{l}\text { Minimum value of Secondary } \\
\text { transmit Power }\end{array}$ & $0 \mathrm{~dB}$ \\
\hline $\begin{array}{l}\text { Maximum value of Secondary } \\
\text { transmit Power }\end{array}$ & $20 \mathrm{~dB}$ \\
\hline
\end{tabular}

\section{SIMULATION RESULTS}

In this section, the results of uncoded BER for secondary transmitter antenna in a CR system is presented with different values of Secondary transmit Power and difference selection weight. The Rayleigh fading channel has been applied in both secondary to secondary and secondary to primary links to calculate the BER. Figure 1 is calculated for the given fitness function for different values of secondary transmit power without any optimization whereas Figure 2 and Figure 3 show the optimized results for BBO and PSO implemented results respectively.

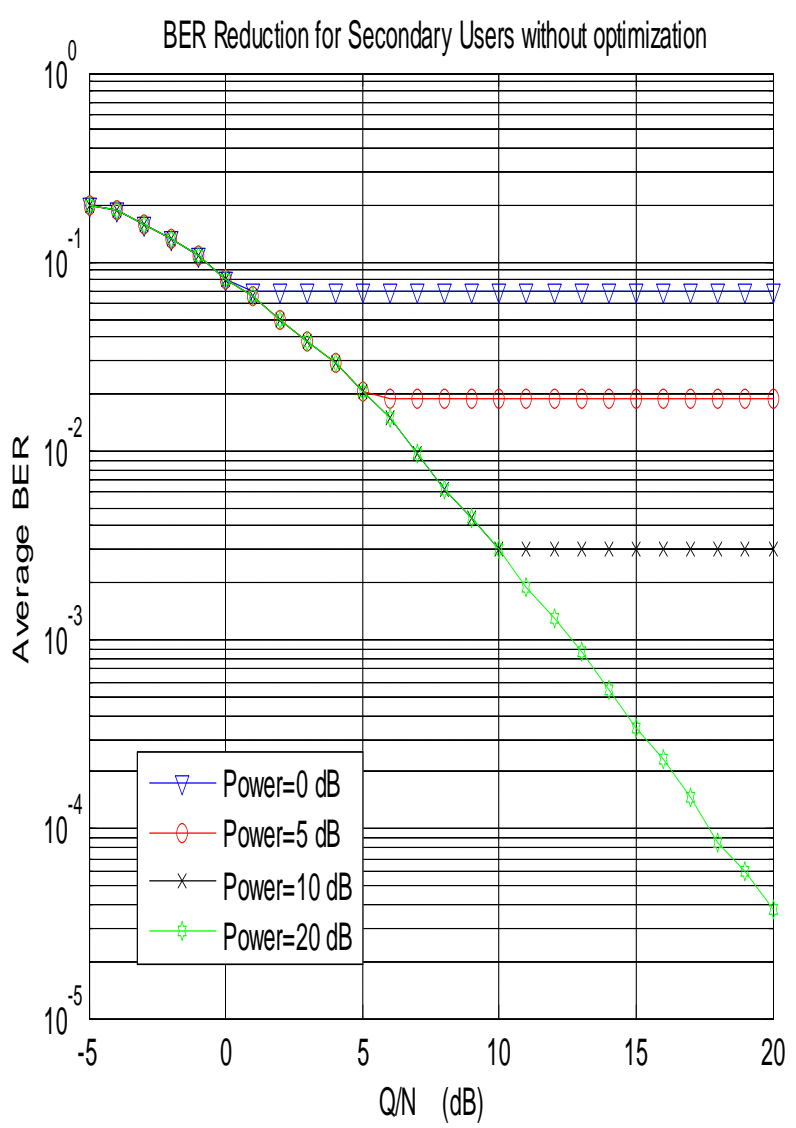

Fig-1: Average BER versus SNR for different values of Transmitted Power without optimization 


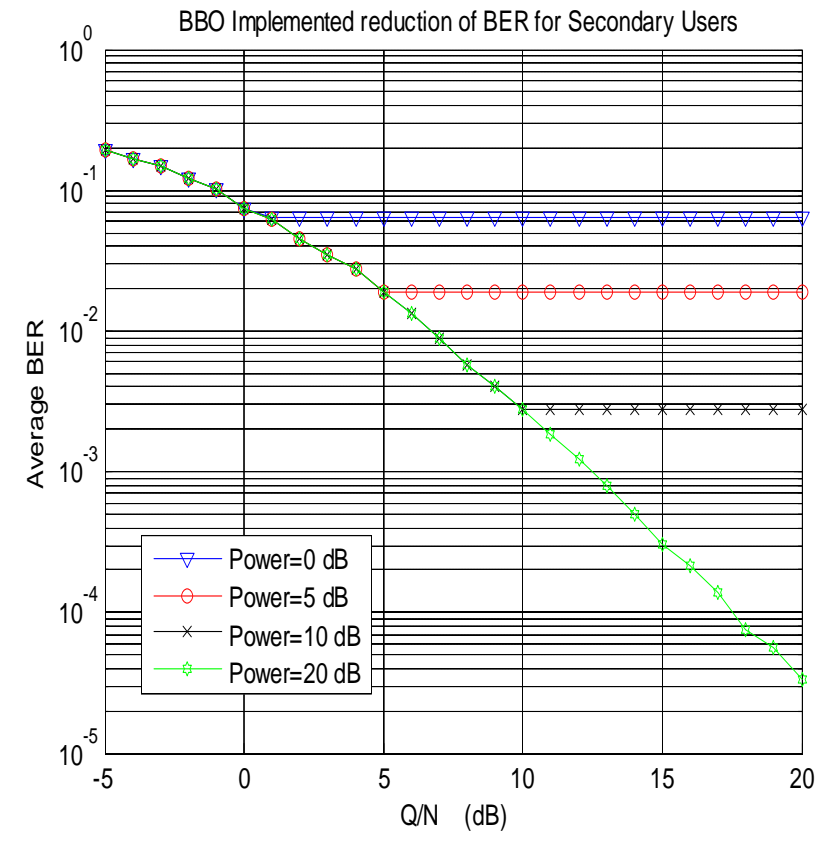

Fig-2: Average BER versus SNR for different values of Transmitted Power using BBO

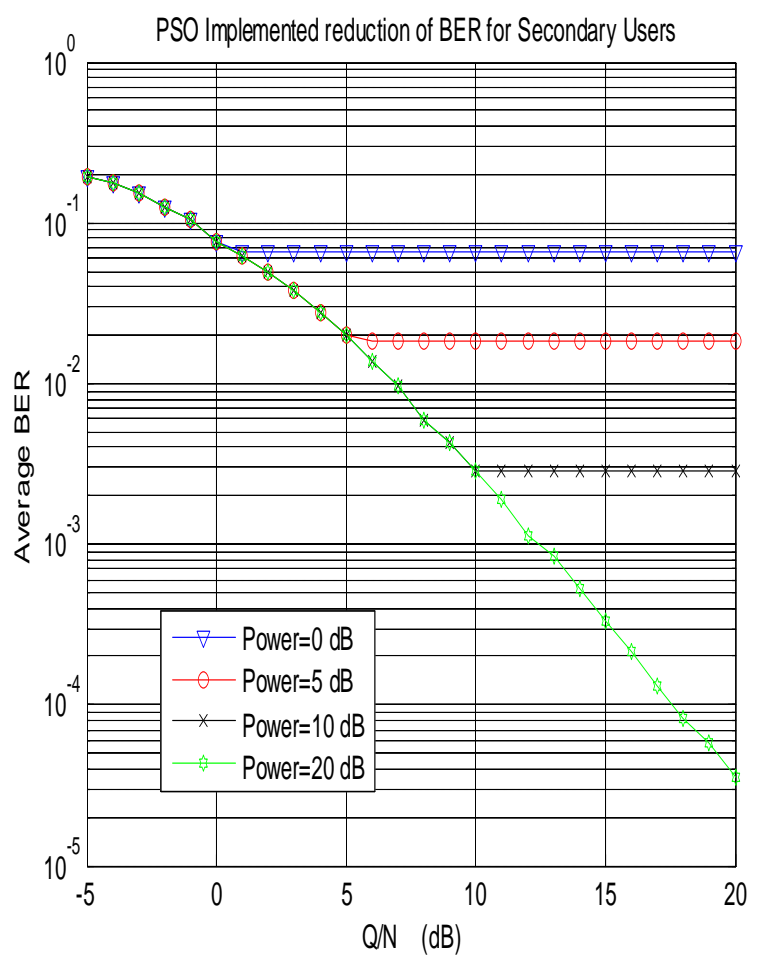

Fig-3: Average BER versus SNR for different values of Transmitted Power using PSO

The results shown above clearly indicate that the BER has improved for all values of SNR in PSO by $5.861 \%$ and in BBO by an average amount of $8.421 \%$ for a constrained secondary power of $5 \mathrm{~dB}$. In particular, the BER for SNR of $15 \mathrm{~dB}$ is $3.406 \times 10^{-4}$ without optimization and it has reduced to $3.291 \times 10^{-4}$ after optimized with PSO and further reduced to $3.053 \times 10^{-4}$ with $\mathrm{BBO}$ method.
Figure 4 shows the simulated results for Average BER versus SNR for different amount of difference selection weight without optimization. Figure 5 and Figure 6 show the graph for BBO and PSO optimized Average BER for the given fitness function. In particular, the BER for SNR at 2 $\mathrm{dB}$ is $7.99 \times 10^{-2}$ for a preselected value of difference selection weight $=0.8$ without any optimization and has been reduced to $7.28 \times 10^{-2}$ with the optimization with $\mathrm{BBO}$ method and further decreased to an amount of $7.52 \times 10^{-2}$ with PSO implementation.

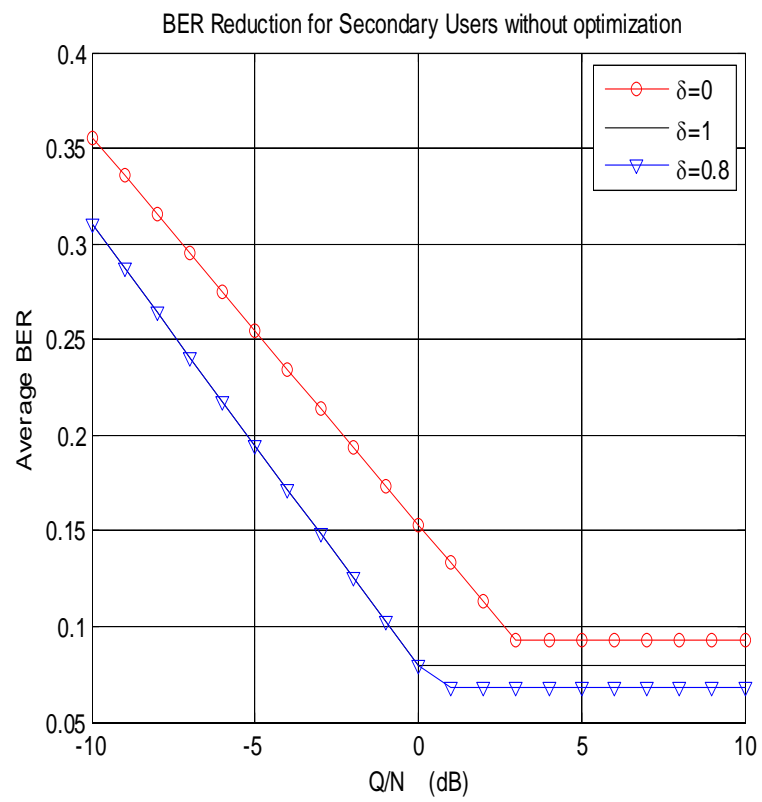

Fig-4: Average BER versus SNR for different values of difference selection weight without optimization

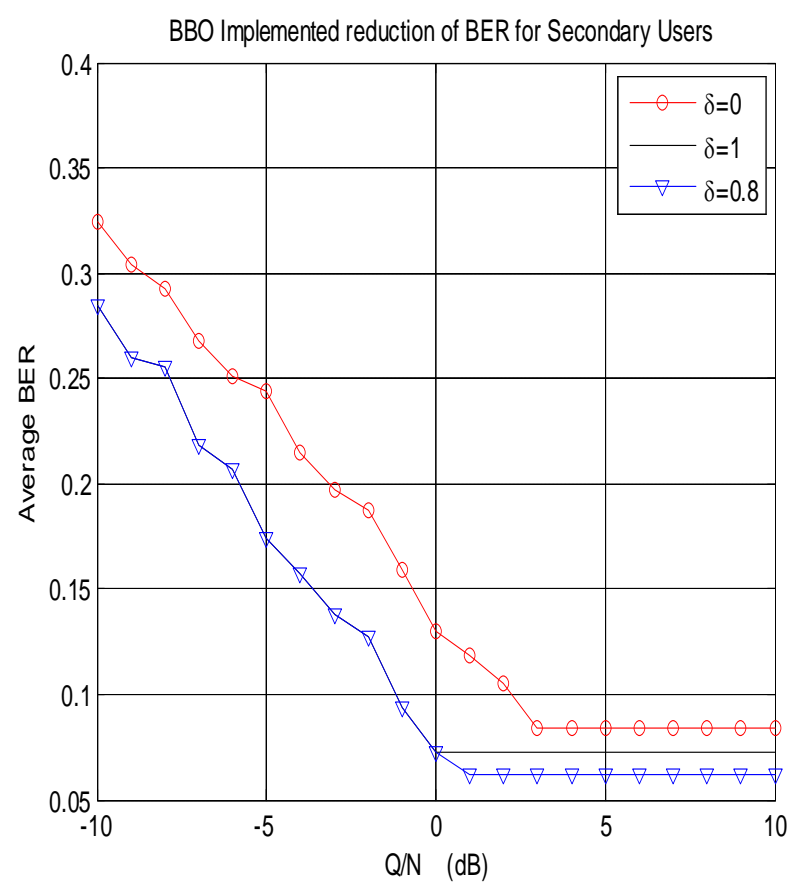

Fig-5: Average BER versus SNR for different values of Difference selection weight using BBO 


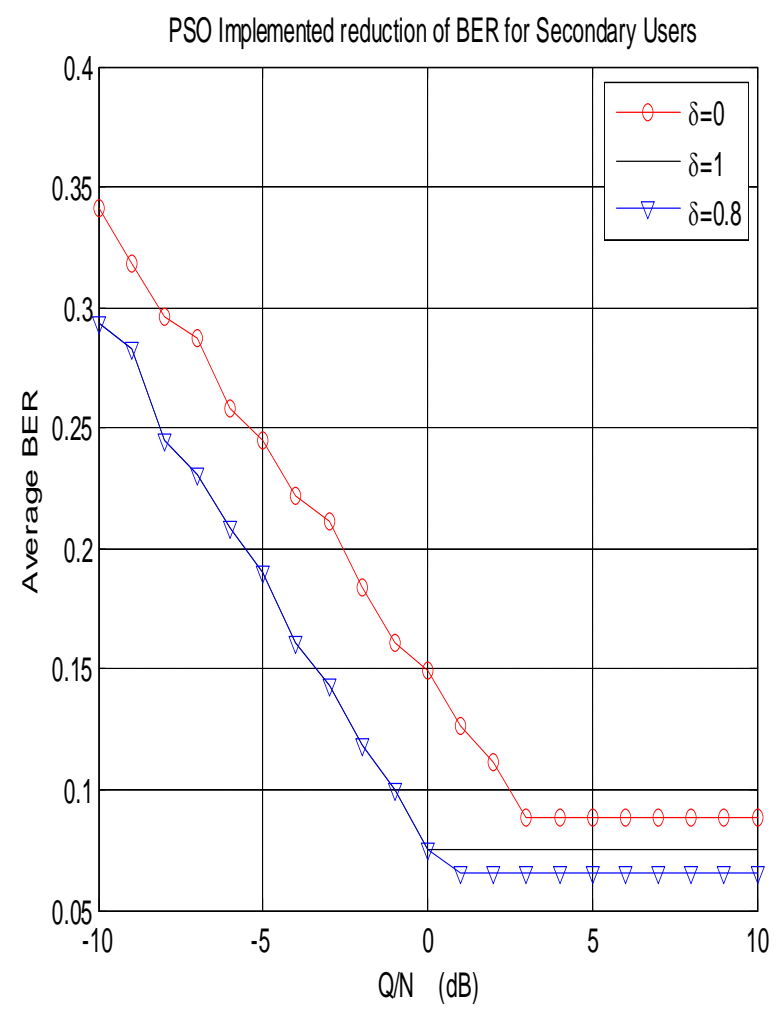

Fig-6: Average BER versus SNR for different values of Difference selection weight using PSO

Table-3 shows the average reduction in BER and the value of time elapsed for the calculation of both optimization methods. It can be observed that in case of $\mathrm{BBO}$, it has taken a time of 0.048169 seconds to reduce the BER whereas in case of PSO, the time elapsed is 0.036195 seconds.

Table-3: Performance comparison for various Optimization methods

\begin{tabular}{|l|l|l|}
\hline $\begin{array}{l}\text { Optimization } \\
\text { Technique }\end{array}$ & $\begin{array}{l}\text { Average Reduced } \\
\text { BER }(\%)\end{array}$ & $\begin{array}{l}\text { Average Time } \\
\text { Elapsed }(\mathrm{s})\end{array}$ \\
\hline BBO & 8.571 & 0.048169 \\
\hline PSO & 5.229 & 0.036195 \\
\hline
\end{tabular}

\section{CONCLUSIONS}

From the obtained results, it can be concluded that the Average BER has been reduced with both proposed algorithms of optimization. For BBO, the BER has been reduced by an average value of $8.571 \%$ in an average time interval of 0.048169 seconds whereas in case of PSO, the BER has decreased up to an average value of $5.229 \%$ in average time of 0.036195 seconds. It seems there exist a trade-off between the two parameters. For any application in CR networks both the BER and Time elapsed are important factors. So in a CR system where data efficiency has high significance like military application, BBO will be preferred because of its better performance in reducing BER. Similarly in systems like vehicular network, where time has high importance over BER, PSO method is more likely to apply.

\section{REFERENCES}

[1]. Dong In Kim, Long Le, Hossain, E., "Resource Allocation for Cognitive Radios in Dynamic Spectrum Access Environment," Cognitive Radio Oriented Wireless Networks and Communications, vol. 1, pp. 1-6, 2008.

[2]. J. G. Proakis, Digital Communication. Fourth Edition, New York, NY: McGraw-Hill, 2001.

[3]. X. Kang, Y. C. liang, H. K. Garg and L. Zhang, "Sensing-Based Spectrum Sharing in Cognative Radio Networks," IEEE Transactions on Vehicular Technology, vol. 58, no. 8, pp. 4649-4654, 2009.

[4]. Y. Wang and J. P. Coon, "BER minimization for cognitive radio systems with difference antenna selection," in IEEE Pacific RimConference on Communication, Computers and Signal Processing, pp. 304-309, 2011.

[5]. H. Wang, J. Lee, S. Kim and D. Hong, "Capacity enhancement of secondary links through spatial diversity in spectrum sharing," IEEE Transactions on Wireless Communications, vol. 9, no. 2, pp. 494-499, 2010.

[6]. Y. Wang and J. Coon, "Difference antenna selection and power allocation for wireless cognative systems" Arxiv preprint arXiv: 1010.0200, 2010. [Online].

[7]. D. Simon, "Biography-based optimization," IEEE Transactions on Evolutionary Computation, Vol. 12, pp. 702-713, Dec 2008.

[8]. X. Hu, Y. Shi and R. Eberhart, "Recent advances in Particle Swarm," Evolutionary Computation, 2004. EC2004. Vol. 1, pp. 90-97, 2004.

[9]. I.F. Akyildiz, W. Y. Lee, M. C. Vuran, and S. Mohant, "Next generation dynamic spectrum access/cognitive radio wireless networks: a survey," Comput.Networks 50(13), pp. 2127-2159, 2006.

[10]. R. Zhang, X. Kang, and Y.-C. Liang, "Protecting primary users in cognitive radio networks: Peak or average interference power constraint?" in IEEE ICC 2009, pp. 1-5, June 2009.

[11]. M. Ergezer, Du Dawei, D. Simon and R. Rarick, "Markov Models for Biogeography-Based Optimization," IEEE Transactions on Systems, Man, and Cybernetics, Volume 41, Issue 1, pp. 299-306, 2011.

[12]. S. Singh, S. Shivangna, ; E. Mittal, "Range Based Wireless Sensor Node Localization Using PSO and BBO and Its Variants," International Conference on Communication Systems and Network Technologies (CSNT), Page 309-315, 2013.

[13]. Ma Haiping, Lin Shengdong, Baogen Jin, "Oppositional Particle Swarm Optimization Algorithm and Its Application to Fault Monitor," Chinese Conference on Pattern Recognition, (CCPR 2009), pp. 1-5, 2009.

[14]. Qinghai Bai, "Analysis of Particle Swarm Optimization Algorithm," Journal of computer and information science, vol. 3, No.1, pp. 180-184, Feb 2010.

[15]. W. H. Press, B. P. Flannery, S. A. Teukolsky, and W. T. Vetterling, Numerical recipes. Cambridge university press Cambridge, 2007. 